\title{
Numerical Radius Perserving Operators on B(H)
}

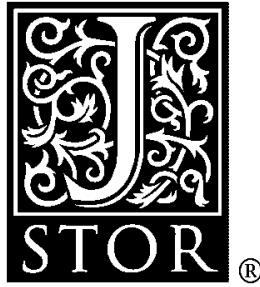

Jor-Ting Chan

Proceedings of the American Mathematical Society, Vol. 123, No. 5. (May, 1995), pp. 1437-1439.

Stable URL:

http://links.jstor.org/sici?sici=0002-9939\%28199505\%29123\%3A5\%3C1437\%3ANRPOO\%3E2.0.CO\%3B2-0

Proceedings of the American Mathematical Society is currently published by American Mathematical Society.

Your use of the JSTOR archive indicates your acceptance of JSTOR's Terms and Conditions of Use, available at

http://www.jstor.org/about/terms.html. JSTOR's Terms and Conditions of Use provides, in part, that unless you have obtained prior permission, you may not download an entire issue of a journal or multiple copies of articles, and you may use content in the JSTOR archive only for your personal, non-commercial use.

Please contact the publisher regarding any further use of this work. Publisher contact information may be obtained at http://www.jstor.org/journals/ams.html.

Each copy of any part of a JSTOR transmission must contain the same copyright notice that appears on the screen or printed page of such transmission.

JSTOR is an independent not-for-profit organization dedicated to and preserving a digital archive of scholarly journals. For more information regarding JSTOR, please contact support@jstor.org. 


\title{
NUMERICAL RADIUS PERSERVING OPERATORS ON $B(H)$
}

\author{
JOR-TING CHAN
}

(Communicated by Palle E. T. Jorgensen)

\begin{abstract}
Let $H$ be a Hilbert space over $\mathbb{C}$ and let $B(H)$ denote the vector space of all bounded linear operators on $H$. We prove that a linear isomorphism $T: B(H) \rightarrow B(H)$ is numerical radius-preserving if and only if it is a multiply of a $C^{*}$-isomorphism by a scalar of modulus one.
\end{abstract}

\section{INTRODUCTION}

Let $H$ be a Hilbert space over $\mathbb{C}$ and let $B(H)$ denote the vector space of all bounded linear operators on $H$. For every $A$ in $B(H)$, the numerical range and the numerical radius of $T$ are defined respectively by

$$
\begin{aligned}
& W(A)=\{\langle A x, x\rangle: x \in H,\|x\|=1\}, \\
& w(A)=\sup \{|\lambda|: \lambda \in W(A)\} .
\end{aligned}
$$

It is well known that $w(\cdot)$ is a norm on $B(H)$ and that this norm is equivalent to the usual operator norm. (See [4, p. 117].) A classical theorem of Kadison [4, Theorem 7] asserts that every linear isomorphism on $B(H)$ which is isometric with respect to the operator norm is a $C^{*}$-isomorphism followed by left multiplication by a fixed unitary operator. A $C^{*}$-isomorphism is a linear isomorphism of $B(H)$ such that $T\left(A^{*}\right)=T(A)^{*}$ for all $A$ in $B(H)$ and $T\left(A^{n}\right)=T(A)^{n}$ for all selfadjoint $A$ in $B(H)$ and all natural number $n$. A description of $C^{*}$-isomorphisms on $B(H)$ can be obtained. First of all we have from [6, Corollary 11] that a $C^{*}$-isomorphism on $B(H)$ is either a ${ }^{*}$ isomorphism or a ${ }^{*}$-anti-isomorphism. Suppose that $T$ is an algebra isomorphism on $B(H)$. Then by [3, Theorem 2], there is an invertible operator $V$ on $H$ such that $T(A)=V A V^{-1}$ for all $A$ in $B(H)$. If we also assume that $T\left(A^{*}\right)=T(A)^{*}$ for all $A$ in $B(H)$, then $V A^{*} V^{-1}=\left(V^{-1}\right)^{*} A^{*} V^{*}$ and hence $\left(V^{*} V\right) A^{*}=A^{*}\left(V^{*} V\right)$ for all $A$ in $B(H)$. It follows that $V^{*} V$ is a scalar multiple of the identity operator $I$. Say $V^{*} V=k I$. As $V^{*} V$ is always a positive operator and $k$ cannot be zero, $k>0$. Let $U=\frac{1}{\sqrt{k}} V$. Then $U$ is unitary and $T(A)=U A U^{*}$ for all $A$ in $B(H)$. For a ${ }^{*}$-anti-isomorphism $T$, it can be shown (e.g., see [5, Remark 2]) that there is a unitary operator $U$ in $B(H)$ such that $T(A)=U A^{t} U^{*}$ for all $A$ in $B(H)$, where $A^{t}$ denotes the transpose

Received by the editors June 15, 1993 and, in revised form, August 2, 1993.

1991 Mathematics Subject Classification. Primary 47B49, 47A12. 
of $A$ relative to a fixed orthonormal basis of $H$. Clearly operators of these two types are $C^{*}$-isomorphisms.

Let us turn to numerical range and numerical radius. Pellegrini [9, Theorem 3.1] proved that an operator $T$ on $B(H)$ is a $C^{*}$-isomorphism exactly when $T$ preserves the "numerical range" of each element in $B(H)$. It should be noted that Pellegrini obtained his result in a general Banach algebra, and his definition of numerical range is different from ours. In fact, for each $A$ in $B(H)$, the "numerical range" of $A$ defined by Pellegrini reduces to the closure of $W(A)$. When the underlying space $H$ is finite-dimensional, $W(A)$ is compact and hence the two sets are identical. Despite the discrepancy we still have that $T$ is a $C^{*}$-isomorphism if and only if $W(T(A))=W(A)$ for every $A$ in $B(H)$. For simplicity we shall call an operator $T$ with the latter property numerical range-preserving. Likewise we say that $T$ is numerical radius-preserving if $w(T(A))=w(A)$ for all $A$ in $B(H)$.

In the finite-dimensional situation, the above result was extended by Li. In [1, Theorem 1] he proved that $T$ is numerical radius-preserving if and only if $T$ is a scalar multiple of a $C^{*}$-isomorphism by a complex number of modulus one. It is immediate that if $T$ is numerical range-preserving, then $T$ is numerical radius-preserving and hence the scalar in question is one. In this note we prove that the conclusion of $\mathrm{Li}$ remains valid without the dimension constraint.

\section{RESUlts}

In what follows $T$ denotes a linear isomorphism on $B(H)$ which is numerical radius-preserving on $B(H)$. We shall prove that $T$ maps the identity mapping $I$ to a scalar multiple of $I$. The scalar is necessarily of modulus one. Multiplying by the complex conjugate of the scalar, we get a numerical radiuspreserving operator $T_{1}$ with an additional property that $T_{1}(I)=I$. The result is concluded by showing that $T_{1}$ is a $C^{*}$-isomorphism.

We begin with a lemma which describes scalar multiples of $I$ in terms of numerical radius. Let $\Lambda=\{\lambda \in \mathbb{C}:|\lambda|=1\}$.

Lemma 1. An operator $A \in B(H)$ is a scalar multiple of $I$ if and only if for every $B \in B(H)$, there is a $\lambda \in \Lambda$ such that $w(A+\lambda B)=w(A)+w(B)$.

Proof. It is clear that if $A$ is a scalar multiple of $I$, then $A$ satisfies the condition. For the converse we borrow the idea from Li and Tsing [2, p. 40]. We first show that elements in $W(A)$ are of constant modulus; it follows then from the convexity of $W(A)([4$, p. 113$])$ that the set is a singleton. Hence $A$ is a scalar multiple of the identity $I$. Now assume that there is an $x$ in $H,\|x\|=1$, and $|\langle A x, x\rangle|<w(A)$. Let $B$ be the orthogonal projection onto the linear span of $x$. Then $w(B)=1$. Fix any $r$ such that $|\langle A x, x\rangle|<r<w(A)$. We can find an $\varepsilon>0$ such that $|\langle A y, y\rangle|<r$ whenever $\|y-x\|<\varepsilon$. In fact $|\langle A y, y\rangle|<r$ if there is a $\lambda \in \Lambda$ such that $\|y-\lambda x\|<\varepsilon$. Suppose that $y \in H,\|y\|=1$, and $\|y-\lambda x\| \geq \varepsilon$ for every $\lambda \in \Lambda$. Then

$$
\varepsilon^{2} \leq\langle y-\lambda x, y-\lambda x\rangle=2-2 \operatorname{Re}\langle y, \lambda x\rangle \text { for every } \lambda \in \Lambda .
$$

It follows that $\langle y, x\rangle \leq 1-\frac{1}{2} \varepsilon^{2}$. Let $k=\min \left\{r+1, w(A)+1-\frac{1}{2} \varepsilon^{2}\right\}$. Then for every $\lambda \in \Lambda$ and $y \in H$ with $\|y\|=1$, we have

$$
\mid\langle(A+\lambda B) y, y|\leq|\langle A y, y\rangle|+|\langle y, x\rangle| \leq k \text {. }
$$

Hence $w(A+\lambda B)<w(A)+w(B)$. 
By the above lemma $T(I)=\lambda I$. Clearly we have $\lambda \in \Lambda$. Let $T_{1}=\bar{\lambda} T$. Then $T_{1}(I)=I$. We need the following definitions. By a state on $B(H)$ we mean as usual a bounded linear functional $\rho$ on $B(H)$ such that $\rho(I)=\|\rho\|=1$. The set $S$ of all states is called the state space of $B(H)$. A bounded linear operator $T: B(H) \rightarrow B(H)$ is said to be state-preserving if its adjoint $T^{\prime}$ satisfies $T^{\prime}(S) \subseteq S$. By [9, Theorem 2.3 and Theorem 3.1], $T$ is a $C^{*}$-isomorphism if and only if it is state-preserving. Let $x$ be a unit vector in $H$. The linear functional $\rho_{x}$ given by

$$
\rho_{x}(A)=\langle A x, x\rangle \text { for every } A \in B(H)
$$

is a state of $B(H)$. States of this form are called vector states.

Lemma 2. The operator $T_{1}$ is state-preserving.

Proof. Let $w^{\prime}$ denote the norm in $B(H)^{\prime}$ dual to the numerical radius. Then $w^{\prime}(\rho) \geq\|\rho\|$ for every $\rho$ in $B(H)^{\prime}$. As $T_{1}$ is numerical radius-preserving, $w^{\prime}\left(T_{1}^{\prime}(\rho)\right)=w^{\prime}(\rho)$ for every $\rho$ in $B(H)^{\prime}$. If $\rho_{x}$ is a vector state, then $w^{\prime}\left(\rho_{x}\right)=$ 1 and hence $\left\|T_{1}^{\prime}\left(\rho_{x}\right)\right\| \leq w^{\prime}\left(T_{1}^{\prime}\left(\rho_{x}\right)\right)=1$. But $T_{1}^{\prime}\left(\rho_{x}\right)(I)=\rho_{x}\left(T_{1}(I)\right)=\rho_{x}(I)=$ 1. It follows that $T_{1}^{\prime}\left(\rho_{x}\right)$ is a state of $B(H)$. By [4, Corollary 4.3.10] the state space is the closed convex hull of the vector states in the weak *-topology. This together with the fact that $T_{1}^{\prime}$ is continuous in the weak *-topology entail that $T_{1}$ is state-preserving.

By Lemma 1 and Lemma 2, we have proved

Theorem. A linear isomorphism $T$ on $B(H)$ is numerical radius-preserving if and only if $T$ is a multiple of a $C^{*}$-isomorphism by a scalar of modulus one.

In [1] $\mathrm{Li}$ also studied a numerical radius-preserving real-linear operator on the selfadjoint elements in $B(H)$. He proved ([1, Theorem 2]) that such an operator is the restriction of a $C^{*}$-isomorphism on $B(H)$ multiplied by \pm 1 . Let us remark that as the numerical radius and the operator norm coincide on selfadjoint operators, this result can alternatively be deduced from [7, Theorem 2].

\section{REFERENCES}

1. C. K. Li, Linear operators preserving the numerical radius of matrics, Proc. Amer. Math. Soc. 99 (1987), 601-608.

2. C. K. Li and N. K. Tsing, Linear operators that preserve the c-numerical range or radius of matrices, Linear and Multilinear Algebra 23 (1988), 27-46.

3. M. Eidelheit, On isomorphisms of rings of linear operators, Studia Math. 9 (1940), 97-105.

4. P. R. Halmos, A Hilbert space problem book, 2nd ed., Springer-Verlag, New York, 1982.

5. A. A. Jafarian and A. R. Sourour, Spectrum-preserving linear maps, J. Funct. Anal. 66 (1986), 255-261.

6. R. V. Kadison, Isometries of operator algebra, Ann. of Math. (2) 54 (1951), 325-338.

7. _ A generalized Schwarz inequality and algebraic invariants for operator algebras, Ann. of Math. (2) 56 (1952), 494-503.

8. R. V. Kadison and J. R. Ringrose, Fundamentals of the theory of operator algebras. I, Academic Press, New York, 1983.

9. V. J. Pellegrini, Numerical range preserving operators on a Banach algebra, Studia Math. 54 (1975), 143-147.

Department of Mathematics, University of Hong Kong, Hong Kong

E-mail address: jtchan@hkucc.bitnet 
http://www.jstor.org

\section{LINKED CITATIONS \\ - Page 1 of 1 -}

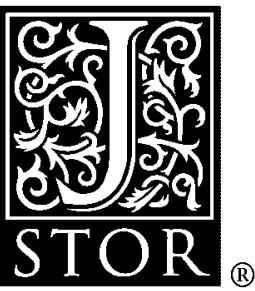

You have printed the following article:

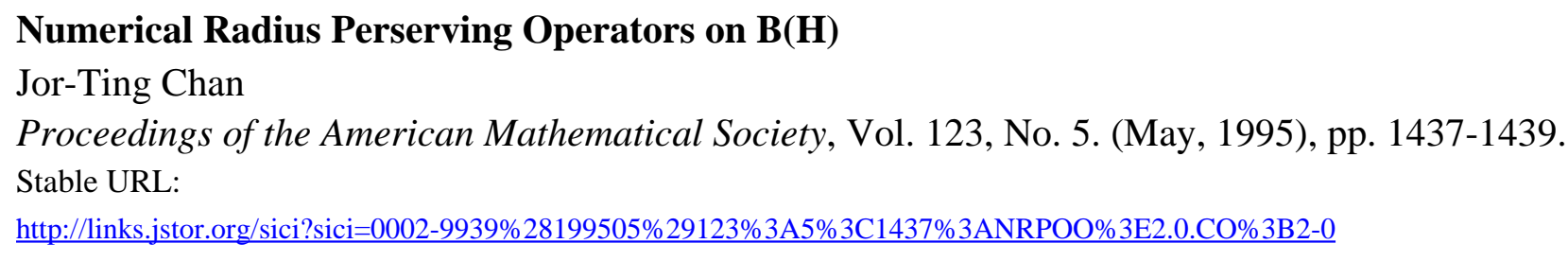

This article references the following linked citations. If you are trying to access articles from an off-campus location, you may be required to first logon via your library web site to access JSTOR. Please visit your library's website or contact a librarian to learn about options for remote access to JSTOR.

\section{References}

\footnotetext{
${ }^{1}$ Linear Operators Preserving the Numerical Radius of Matrices

Chi-Kwong Li

Proceedings of the American Mathematical Society, Vol. 99, No. 4. (Apr., 1987), pp. 601-608.

Stable URL:

http://links.jstor.org/sici?sici=0002-9939\%28198704\%2999\%3A4\%3C601\%3ALOPTNR\%3E2.0.CO\%3B2-P
}

NOTE: The reference numbering from the original has been maintained in this citation list. 